\title{
Multiproduct Uniform Polar Quantizer
}

\author{
Milan R. DINCIC, Zoran H. PERIC \\ Faculty of Electronic Engineering, University of Nis, Aleksandra Medvedeva 14, 18000 Nis, Serbia \\ mdincha@hotmail.com, zoran.peric@elfak.ni.ac.rs
}

\begin{abstract}
The aim of this paper is to reduce the complexity of the unrestricted uniform polar quantizer (UUPQ), keeping its high performances. To achieve this, in this paper we propose the multiproduct uniform polar quantizer (MUPQ), where several consecutive magnitude levels are joined in segments and within each segment the uniform product quantization is performed (i.e. all levels within one segments have the same number of phase levels). MUPQ is much simpler for realization than UUPQ, but it achieves similar performances as UUPQ. Since MUPQ has low complexity and achieves much better performances than the scalar uniform quantizer, it can be widely used instead of scalar uniform quantizers to improve performances, for any signal with the Gaussian distribution.
\end{abstract}

\section{Keywords}

Unrestricted uniform polar quantization, multiproduct uniform polar quantization, polar coordinates, Gaussian distribution

\section{Introduction}

Digital transmission and processing are dominant nowadays, while the most signals are analog. Therefore, an inevitable part of almost all modern telecommunication systems is $\mathrm{A} / \mathrm{D}$ (analog-to-digital) converter. The main part of $\mathrm{A} / \mathrm{D}$ converters is a quantizer. The quality of the digitized signal mostly depends on the quantizer. Also, with the appropriate design of the quantizer, the compression of signals can be achieved, which is very important since resources for the transmission and storage of signals (channel bandwidth and memory space) are limited. Therefore, the designing of quantizers is very important and topical subject.

There are two main types of quantizers [1], [2]: scalar quantizers (where each sample of the signal is separately quantized) and vector quantizers (where several samples are jointly quantized; for example, if $n$ samples are jointly quantized, we can say that they represent an $n$-dimensional vector in $n$-dimensional vector space, therefore we have an $n$-dimensional vector quantizer). Vector quantizers have much better performances (i.e. they can achieve much higher SQNR (signal-to-quantization noise ratio) for the same bit-rate) than scalar quantizers [2]. On the other hand, vector quantizers have one drawback: high complexity, which exponentially increases with the increasing of the dimension $n$. Therefore, the most used vector quantizers are two-dimensional quantizers, which are the simplest of all vector quantizers, but they still have much better performances than scalar quantizers. Almost all signals of interest are random and can be described with some probability density function (pdf). A lot of signals can be modeled with the Gaussian pdf. For signals with the Gaussian pdf, it is easier to design two-dimensional vector quantizer in the polar coordinates (magnitude $r$ and phase $\phi$ ) than in the Cartesian coordinates. Such quantizers, designed in the polar coordinates, are called polar quantizers.

There are two main types of polar quantizers: restricted (also called product) and unrestricted. In product polar quantizers, the number of phase levels is the same for all magnitude levels, while in unrestricted polar quantizers the numbers of phase levels are different for different magnitude levels, i.e. the number of phase levels are optimized for each magnitude level. Unrestricted polar quantizers have better performances than product polar quantizers, but they are more complex. In polar quantizers, the magnitude $r$ can be quantized in different ways (using uniform or nonuniform quantization), while the phase $\phi$ is always quantized using the uniform quantization since it has the uniform distribution.

Polar quantization has been considered in many papers. In [3], [4] unrestricted polar quantizers were analyzed, using the optimal companding function for the quantization of the magnitude $r$. In [5], [6] the product uniform polar quantization was considered. The product polar quantizer with the companding function optimal for scalar but not for polar quantization, was considered in [7]. Embedded product and unrestricted polar quantizers were considered in [8]. Product polar quantizers with A-law companding function were analyzed in [9] with the application for audio signals. In [10], [11] product polar quantizers with $\mu$-law companding function were considered. The solution in [11] is compatible with the ITU-T G.711 standard. Unrestricted polar quantizers with square cells were analyzed in [12], applying $\mu$-law companding function for the quantization of the magnitude $r$.

The unrestricted uniform polar quantizer (UUPQ) is the polar quantizer with uniformly quantized magnitude $r$, where the optimization of numbers of phase levels is done 
for each magnitude levels. It was analyzed in [13], [14]. It is two-dimensional polar counterpart of the scalar uniform quantizer. UUPQ can achieve much better performances (i.e. much higher SQNR for the same bit-rate) than the scalar uniform quantizer. UUPQ can be used instead of the scalar uniform quantizer (which is widely used), to improve performances. However, UUPQ has high complexity, since numbers of phase levels for all magnitude levels should be calculated and stored in memory.

The aim of this paper is to reduce the complexity of UUPQ, keeping its good performances. Therefore, in this paper we propose the multiproduct uniform polar quantizer (MUPQ), where several magnitude levels are joined into segments. Within each segment the product polar quantization is done, i.e. all magnitude levels in one segment have the same number of phase levels. Therefore, for MUPQ we have to calculate numbers of phase levels for each segment, not for each magnitude level (which is the case in UUPQ). Since the number of segments is much smaller than the number of magnitude levels, it follows that for MUPQ we have to calculate much smaller number of parameters than for UUPQ, thus MUPQ is much simpler for realization than UUPQ. Furthermore, we will show that performances of MUPQ are very close to performances of UUPQ. Therefore, the main contribution of this paper is the designing of MUPQ, which is much simpler for realization than UUPQ, but which can achieve excellent performances, very close to performances of UUPQ.

The comparison between MUPQ and PUPQ (product uniform polar quantizer) [5], [6] is also presented. It is shown that MUPQ is better solution since MUPQ is slightly more complex than PUPQ but MUPQ achieves higher SQNR compared to PUPQ. Slight increase of complexity provides appreciable increasing of SQNR.

The asymptotic analysis is usually used for the designing of polar quantizers [3-14]. Hence, the asymptotic analysis will be applied in this paper. We will consider medium and high bit-rates (roughly speaking, higher than 4 bps (bits-per-sample)), since the asymptotic analysis is valid for these bit-rates.

Simulations are done in MATLAB both for UUPQ and MUPQ. Simulation and theoretical results are matched very well, which proves the correctness of the developed theory.

Theory is also proven by the experiment performed on the speech signal, for MUPQ, PUPQ and UUPQ. We use the speech signal since it can be modeled with the Gaussian distribution very well [1].

This paper is organized in the following way. Polar quantizers are defined in Section 2. In Section 3, the analysis of performances of UUPQ is presented shortly. The designing of MUPQ, which is the main contribution of this paper, is presented in Section 4. Numerical results and discussion are given in Section 5. Section 6 concludes the paper.

\section{The Definition of Polar Quantizer}

Let's consider a signal with the Gaussian distribution, which is defined with the probability density function (pdf) $f(x)=\frac{1}{\sqrt{2 \pi} \sigma} \exp \left(-x^{2} /\left(2 \sigma^{2}\right)\right)$, where $\sigma^{2}$ denotes the power (variance) of the signal. The design of quantizers is usually done for the unit variance $\sigma^{2}=1$, hence we will use this approach in this paper. For the designing of two-dimensional quantizers, the joint probability density function of two consecutive samples $x_{1}$ and $x_{2}$ should be used. In Cartesian coordinates, the joint pdf is defined as $f\left(x_{1}, x_{2}\right)=\exp \left(-\left(x_{1}^{2}+x_{2}^{2}\right) / 2\right) /(2 \pi)$. Let's define polar coordinates: magnitude $r=\sqrt{x_{1}^{2}+x_{2}^{2}}$ and phase $\phi=\arctan \left(x_{2} / x_{1}\right)$. The joint pdf in polar coordinates becomes $f(r, \phi)=r \exp \left(-r^{2} / 2\right) /(2 \pi)$ [12]. We can see that $f(r, \phi)$ depends only on $r$ but not on $\phi$. The pdf of the magnitude $r$ is:

$$
f_{r}(r)=\int_{0}^{2 \pi} f(r, \phi) d \phi=r e^{-r^{2} / 2}
$$

which is the pdf of the Rayleigh distribution. The pdf of the phase $\phi$ is $f_{\phi}(\phi)=\int_{0}^{+\infty} f(r, \phi) d r=\frac{1}{2 \pi}$. We can see that the phase $\phi$ has the uniform distribution, which means that the two-dimensional source of information is circularlysymmetric. One often used approach of the designing of vector quantizers is the geometric principle [15]. According to this principle, quantization cells are deployed on contours where the joint pdf is constant. Since the joint pdf of the Gaussian source in polar coordinates $f(r, \phi)$ depends only on $r$, it follows that $f(r, \phi)$ is constant where $r=$ const., i.e. contours where $f(r, \phi)$ is constant are concentric circles. Applying the geometric principle, the two-dimensional quantizer for the Gaussian source is much easier to design in polar than in Cartesian coordinates, by deploying cells on concentric circles. Quantizers designed in polar coordinates are called polar quantizers.

Let's define some parameters of polar quantizers: $N$ denotes the total number of quantization cells, $r_{\max }$ denotes the maximal magnitude, $L$ denotes the number of magnitude levels, $r_{i}, i=0, \ldots, L$ denote thresholds and $m_{i}$, $i=1, \ldots, L$ denote representation levels for the quantization of the magnitude $r$. On each magnitude level the uniform quantization of the phase $\phi$ is done, since the phase $\phi$ has the uniform distribution. Let $P_{i}$ denote the number of phase levels on the $i$-th magnitude level. It holds that $\sum_{i=1}^{L} P_{i}=N$. Let $\phi_{i, j}=j 2 \pi / P_{i}, \quad j=0, \ldots, P_{i}$ denote thresholds and $\psi_{i, j}=(j-1 / 2) 2 \pi / P_{i}, j=1, \ldots, P_{i}$ denote representation levels for the quantization of the phase $\phi$ for the $i$-th magnitude level. 
Within each cell there is one representation point. Let's consider an arbitrary quantization cell $S_{i, j}=$ $\left\{(r, \phi) \mid r_{i-1} \leq r<r_{i} \wedge \phi_{i, j-1} \leq \phi<\phi_{i, j}\right\}, i=1, \ldots, L, j=$ $1, \ldots, P_{i}$. Within this cell there is the representation point $\left(m_{i}, \psi_{i, j}\right)$. All points $(r, \phi)$ from the cell $S_{i, j}$ are mapped to the representation point $\left(m_{i}, \psi_{i, j}\right)$.

There are two basic types of polar quantizers: unrestricted (where numbers of phase levels are different for different magnitude levels, i.e. the optimization of the number of phase levels is done for each magnitude level) and restricted (also called product, where the numbers of phase levels are the same for all magnitude levels, i.e. $\left.P_{1}=P_{2}=\ldots=P_{L}\right)$.

During the quantization process, an irreversible error is made, which is expressed by the distortion. The total distortion $D$ is equal to the sum of the granular distortion $D_{\mathrm{g}}$ (which is made in the area $0 \leq r \leq r_{\max }$ ) and the overload distortion $D_{\text {ov }}$ (which is made in the area $r>r_{\max }$ ), i.e. $D=D_{\mathrm{g}}+D_{\mathrm{ov}}$. Distortions in this paper will be defined per one dimension. It is usual that performances of vector quantizers are defined per one dimension, to simplify comparison of performances of vector quantizers with different dimensions. The quality of the quantized signal is defined with SQNR (signal-to-quantization noise ratio), which is defined as:

$$
\mathrm{SQNR}[\mathrm{dB}]=10 \log _{10}(1 / D) .
$$

The bit-rate (the average number of bits required for the coding of one sample) is defined as:

$$
R=\frac{1}{2} \log _{2} N[\mathrm{bps}] .
$$

\section{Unrestricted Uniform Polar Quantizer (UUPQ)}

This is the unrestricted polar quantizer where the uniform quantization of the magnitude $r$ is performed, i.e. the magnitude range $\left[0, r_{\max }\right]$ is uniformly divided into $L$ intervals. Thresholds for the uniform quantization of the magnitude $r$ are defined as $r_{i}=i r_{\max } / L, i=0, \ldots, L$, while representation levels are defined as $m_{i}=(i-1 / 2) r_{\max } / L$, $i=1, \ldots, L$. Let $\Delta_{r}=r_{i}-r_{i-1}=r_{\max } / L$ denotes the stepsize for the uniform quantization of the magnitude $r$.

UUPQ was already analyzed in [13], [14]. We will recall some results from [13], [14]. The expression for the number of magnitude levels was derived in [14]:

$$
L=r_{\max } \sqrt{\frac{N}{6 \pi \sqrt{3}}} \cdot\left(1-e^{-r_{\max }^{2} / 6}\right)^{-3 / 4} .
$$

In [13], the following expression for the number of phase levels on the $i$-th magnitude level was presented: $P_{i}=N r_{\max } \sqrt[3]{m_{i}^{2} f_{r}\left(m_{i}\right)}\left(L \int_{0}^{r_{\max }} \sqrt[3]{r^{2} f_{r}(r)} d r\right)^{-1}$. For $f_{r}(r)$ defined with (1), it follows that:

$$
P_{i}=\sqrt{\frac{2 \pi N}{\sqrt{3}}} \frac{m_{i} \exp \left(-m_{i}^{2} / 6\right)}{\sqrt[4]{\left(1-e^{-r_{\max }^{2} / 6}\right)}}, i=1, \ldots, L .
$$

The expression for the granular distortion was derived in [14]:

$$
D_{g}=\frac{\sqrt{3} \pi}{2 N}\left(1-e^{-r_{\max }^{2} / 6}\right)^{3 / 2}
$$

However, the analysis of UUPQ presented in [13] and [14] was incomplete and not quite correct from the following reasons: in [13] the overload distortion was neglected and the optimization of $r_{\max }$ was not done (instead of that, the value of $r_{\max }$ for the scalar uniform quantizer was used); in [14] the expression for the overload distortion was not given and the method for calculation of $r_{\max }$ was not explained. Due to these reasons, performances of UUPQ calculated using the analysis from [13], [14] is not accurate enough. Since we need accurate performances of UUPQ, we will present some new results for UUPQ (calculation of the overload distortion and the optimization of $\left.r_{\max }\right)$ which are missing in [13] and [14].

Using the similar procedure as for the granular distortion in [13], we can define the overload distortion as: $D_{o v}=\frac{1}{2} \int_{r_{\max }}^{+\infty}\left[\left(r-m_{L}\right)^{2}+r m_{L} \pi^{2} /\left(3 P_{L}^{2}\right)\right] f_{r}(r) d r$.

The

article $1 / 2$ on the beginning of this expression denotes the fact that $D_{o v}$ is defined per one dimension. Using approximation $m_{L} \approx r_{\max }$, which is valid for the asymptotic analysis, it is obtained that:

$$
D_{o v}=\frac{1}{2} \int_{r_{\max }}^{+\infty}\left[\left(r-r_{\max }\right)^{2}+\frac{r r_{\max } \pi^{2}}{3 P_{L}^{2}}\right] f_{r}(r) d r .
$$

Using (5) for $P_{L}$, the following final expression for the overload distortion is obtained:

$$
D_{o v}=X+\frac{\sqrt{3} \pi e^{r_{\max }^{2} / 3}}{12 N r_{\max }} \sqrt{\left(1-e^{-r_{\max }^{2} / 2}\right)\left(1-e^{-r_{\max }^{2} / 6}\right)} \cdot Y
$$

where

$$
\begin{aligned}
& X=\frac{1}{2}\left(2 e^{-r_{\max }^{2} / 2}-\sqrt{2 \pi} r_{\max } \operatorname{erfc}\left(r_{\max } / \sqrt{2}\right)\right), \\
& Y=r_{\max } e^{-r_{\max }^{2} / 2}+\sqrt{\pi / 2} \operatorname{erfc}\left(r_{\max } / \sqrt{2}\right) .
\end{aligned}
$$

The total distortion $D$ is obtained by summing expressions (6) and (8). SQNR and the bit-rate $R$ are calculated using (2) and (3). 
Now, we will shortly summarize the design process. The parameter $N$ is defined in advance, i.e. $N$ is known on the beginning of the design process. Optimal values of other parameters $\left(r_{\max }, L\right.$ and $\left.P_{i}\right)$ should be found during the design process, in the following way. The parameter $r_{\max }$ should be found firstly. Based on (6) and (8), we can see that the total distortion $D$ depends only on one unknown parameter $r_{\max }$. Optimal value of $r_{\max }$ is obtained by the minimization of the $D$, i.e. by numerical solving of the equation $\left(d D / d r_{\max }\right)=0$. When $r_{\max }$ is found, we can calculate all other unknown parameters: $L$ is calculated using (4); after that thresholds and representation levels are calculated as $r_{i}=i r_{\max } / L$ and $m_{i}=(i-1 / 2) r_{\max } / L$; finally, values for $P_{i}, i=1, \ldots, L$ are calculated using (5).

Numerical values for UUPQ are given in Tab. 1. Simulation of UUPQ is done in MATLAB. In Tab. 1 we present values of SQNR obtained by the theory $\left(\mathrm{SQNR}_{\mathrm{th}}\right)$ and by the simulation $\left(\mathrm{SQNR}_{\text {sim }}\right)$. We can see that values of SQNR obtained by the theory and by the simulation are matched very well, which proves the previously developed theory.

\begin{tabular}{|c|c|c|c|c|}
\hline$R$ [bps] & 5 & 6 & 7 & 8 \\
\hline$r_{\max }$ & 3.55 & 4.00 & 4.42 & 4.82 \\
\hline$L$ & 22 & 47 & 102 & 219 \\
\hline SQNRth $[\mathrm{dB}]$ & 26.28 & 32.06 & 37.95 & 43.90 \\
\hline SQNRsim $[\mathrm{dB}]$ & 26.25 & 32.05 & 37.92 & 43.89 \\
\hline
\end{tabular}

Tab. 1. Numerical results for UUPQ.

\begin{tabular}{|c|c|c|c|c|}
\hline$R$ [bps] & 5 & 6 & 7 & 8 \\
\hline SQNR [dB] & 24.57 & 29.83 & 35.13 & 40.34 \\
\hline
\end{tabular}

Tab. 2. SQNR for the scalar uniform quantizer [1].

For the purpose of comparison, values of SQNR for the scalar uniform quantizer for different values of $R$ are presented in Tab. 2. These values are taken from [1]. We can see that UUPQ has much better performances (i.e. much higher SQNR) than the corresponding uniform scalar quantizer, for the same bit-rate $R$. Due to its very good performances, UUPQ could be very important in many applications. However, UUPQ has one drawback: high complexity. Namely, due to the fact that the numbers of phase levels $P_{i}$ are different for different magnitude levels, we have to calculate and store parameters $P_{i}$ for all magnitude levels, which increases complexity and requires large memory space, both in transmitter and receiver.

In the aim to decrease the complexity, but also to keep good performances in the same time, we will present the multiproduct uniform polar quantizer (MUPQ) in the next section.

\section{Multiproduct Uniform Polar Quantizer}

The meaning of parameters $N, r_{\max }, L, r_{i}, m_{i}$, and $\Delta_{r}$ is the same as in UUPQ. In MUPQ, the uniform quan- tization of the magnitude $r$ is performed (i.e. the magnitude range $\left[0, r_{\max }\right]$ is uniformly divided into $L$ magnitude intervals) and the uniform quantization of the phase $\phi$ is done for each magnitude interval, as in the previously described UUPQ. But, in MUPQ, $L_{0}$ consecutive magnitude intervals are joined into segments. There are $K=L / L_{0}$ segments. Within one segment, the product polar quantization is performed, which means that all magnitude intervals within one segment have the same number of phase levels. Since this polar quantizer consists of $K$ product uniform polar quantizers, it is called 'multiproduct'. Let $M_{j}$, $j=1, \ldots, K$, denote the number of phase levels on magnitude intervals within the $j$-th segment. It holds that $\sum_{j=1}^{K} M_{j}=N / L_{0}=N K / L$ Let $t_{j}=j r_{\max } / K$, $j=1, \ldots, K$, denote borders between segments. Let $r_{j, l}=t_{j-1}+l \Delta_{r}, j=1, \ldots, K, l=0, \ldots, L_{0}$ denote thresholds and $\quad m_{j, l}=t_{j-1}+(l-1 / 2) \Delta_{r}, \quad j=1, \ldots, K, \quad l=1, \ldots, L_{0}$ denote representation levels for the quantization of the magnitude $r$ in the $j$-th segment. Parameters $N$ and $K$ are given in advance, i.e. they are known at the beginning of the designing process. Optimal values of other parameters should be found through the designing process.

The total distortion $D$ is equal to the sum of the granular $D_{g}$ and overload $D_{o v}$ distortions. The granular distortion $D_{g}$ can be written as $D_{g}=D_{g 1}+D_{g 2}$. It was shown in [13] that $D_{g 1}=\frac{1}{2} \sum_{i=1}^{L} \int_{r_{i-1}}^{r_{i}}\left(r-m_{i}\right)^{2} f_{r}(r) d r$. For the asymptotic analysis it holds that $D_{\mathrm{g} 1}=$ $=\frac{1}{2} \sum_{i=1}^{L} f_{r}\left(m_{i}\right) \int_{r_{i-1}}^{r_{i}}\left(r-m_{i}\right)^{2} d r$. Since $r_{i-1}=m_{i}-\Delta_{r} / 2$ and $r_{i}=m_{i}+\Delta_{r} / 2$, it follows that $D_{g 1}=$ $(1 / 2) \cdot \sum_{i=1}^{L} f_{r}\left(m_{i}\right) \Delta_{r}^{3} / 12=\left(\Delta_{r}^{2} / 24\right) \cdot \sum_{i=1}^{L} f_{r}\left(m_{i}\right) \Delta_{r}$.

Changing summation with integration, it is obtained that $D_{g 1}=\left(\Delta_{r}^{2} / 24\right) \int_{0}^{r_{\max }} f_{r}(r) d r$. Solving this integral for $f_{r}(r)$ defined with (1), it is obtained that:

$$
D_{g 1}=\frac{r_{\max }^{2}}{24 L^{2}}\left(1-\exp \left(-r_{\max }^{2} / 2\right)\right) \text {. }
$$

Based on [13], $D_{g 2}$ can be expressed as: $D_{g 2}=$ $\left(\pi^{2} / 6\right) \sum_{j=1}^{K} \sum_{l=1}^{L_{0}} \int_{r_{j, l-1}}^{r_{j, l}}\left(r m_{j, l} / M_{j}^{2}\right) f_{r}(r) d r$. Applying the asymptotic analysis, it follows that $D_{g 2}=\left(\pi^{2} / 6\right)$ . $\sum_{j=1}^{K}\left(1 / M_{j}^{2}\right) \sum_{l=1}^{L_{0}} m_{j, l} f_{r}\left(m_{j, l}\right) \int_{r_{j, l-1}}^{r_{j, l}} r d r$. Since $\int_{r_{j, l-1}}^{r_{j, l}} r d r$ $=\left(r_{j, l}+r_{j, l-1}\right)\left(r_{j, l}-r_{j, l-1}\right) / 2=m_{j, l} \Delta_{r}$, we obtain that:

$$
D_{g 2}=\frac{\pi^{2}}{6} \sum_{j=1}^{K} \frac{1}{M_{j}^{2}} \sum_{l=1}^{L_{0}} m_{j, l}^{2} f_{r}\left(m_{j, l}\right) \Delta_{r} .
$$


Changing summation with integration, it follows that:

$$
D_{g 2}=\frac{\pi^{2}}{6} \sum_{j=1}^{K} \frac{1}{M_{j}^{2}} \int_{t_{j-1}}^{t_{j}} r^{2} f_{r}(r) d r=\frac{\pi^{2}}{6} \sum_{j=1}^{K} \frac{I_{j}}{M_{j}^{2}}
$$

where

$$
\begin{aligned}
I_{j}= & \int_{t_{j-1}}^{t_{j}} r^{2} f_{r}(r) d r=-\left(2+\frac{j^{2} r_{\text {max }}^{2}}{K^{2}}\right) \exp \left(-\frac{j^{2} r_{\text {max }}^{2}}{2 K^{2}}\right) \\
& +\left(2+\frac{(j-1)^{2} r_{\text {max }}^{2}}{K^{2}}\right) \exp \left(-\frac{(j-1)^{2} r_{\text {max }}^{2}}{2 K^{2}}\right)
\end{aligned}
$$

The expression for the granular distortion becomes $D_{g}=$ $r_{\max }^{2} /\left(24 L^{2}\right) \cdot\left(1-\exp \left(-r_{\max }^{2} / 2\right)\right)+\left(\pi^{2} / 6\right) \sum_{j=1}^{K} I_{j} / M_{j}^{2}$.

To find optimal values of $M_{j}$, minimization of $D_{g}$ will be done with the constraint that $\sum_{j=1}^{K} M_{j}=N K / L$. Function $G=D_{g}+\lambda\left(\sum_{j=1}^{K} M_{j}-N K / L\right)$ is formed, where $\lambda$ is the Lagrange multiplier. Solving the equation $\left(\partial G / \partial M_{j}\right)=0$, it is obtained that $M_{j}=\sqrt[3]{\pi^{2} /(3 \lambda)} \cdot \sqrt[3]{I_{j}}$. Since $\quad \sum_{q=1}^{K} M_{q} \equiv \sqrt[3]{\pi^{2} /(3 \lambda)} \cdot \sum_{q=1}^{K} \sqrt[3]{I_{q}}=N K / L, \quad$ it follows that $\sqrt[3]{\pi^{2} /(3 \lambda)}=N K /\left(L \cdot \sum_{q=1}^{K} \sqrt[3]{I_{q}}\right)$. Therefore, the following expression is obtained:

$$
M_{j}=\frac{N \cdot K}{L} \cdot \frac{\sqrt[3]{I_{j}}}{\sum_{q=1}^{K} \sqrt[3]{I_{q}}}, j=1, \ldots, K
$$

Putting (14) into (12), it follows that:

$$
D_{g 2}=\frac{\pi^{2}}{6} \frac{L^{2}}{N^{2} K^{2}}\left(\sum_{q=1}^{K} \sqrt[3]{I_{q}}\right)^{3}
$$

The granular distortion $D_{g}$ can be written as $D_{g}=C_{1} / L^{2}+C_{2} L^{2}$, where $C_{1}=\left(r_{\max }^{2} / 24\right)\left(1-\exp \left(-r_{\max }^{2} / 2\right)\right)$ and $C_{2}=\pi^{2} /\left(6 N^{2} K^{2}\right) \cdot\left(\sum_{q=1}^{K} \sqrt[3]{I_{q}}\right)^{3}$. Solving the equation $\left(\partial D_{g} / \partial L\right)=0$, it is obtained that:

$$
L=\sqrt[4]{\frac{C_{1}}{C_{2}}}=\sqrt{\frac{r_{\max } N K}{2 \pi}} \sqrt[4]{\frac{1-e^{-r_{\max }^{2} / 2}}{\left(\sum_{q=1}^{K} \sqrt[3]{I_{q}}\right)^{3}}} .
$$

Putting (16) into (14), it follows that:

$$
M_{j}=\frac{\sqrt{2 \pi N K / r_{\max }} \cdot \sqrt[3]{I_{j}}}{\sqrt[4]{\left(1-e^{-r_{\max }^{2} / 2}\right)\left(\sum_{q=1}^{K} \sqrt[3]{I_{q}}\right)}}, j=1, \ldots, K .
$$

Putting $L=\sqrt[4]{C_{1} / C_{2}}$ into $D_{g}=C_{1} / L^{2}+C_{2} L^{2}$, the following final expression for the granular distortion is obtained:

$$
D_{g}=2 \sqrt{C_{1} C_{2}}=\frac{r_{\max } \pi}{6 N K} \sqrt{1-e^{-r_{\max }^{2} / 2}}\left(\sum_{q=1}^{K} \sqrt[3]{I_{q}}\right)^{3 / 2} .
$$

Based on (7), the overload distortion is defined as $D_{o v}=$ $\frac{1}{2} \int_{r_{\max }}^{+\infty}\left[\left(r-r_{\max }\right)^{2}+r r_{\max } \pi^{2} /\left(3 M_{K}^{2}\right)\right] f_{r}(r) d r$. Using

for $M_{K}$, the following final expression for the overload distortion is obtained:

$$
D_{o v}=X+\frac{r_{\max }^{2} \pi^{2} \sqrt{1-e^{-r_{\max }^{2} / 2}}}{12 N K\left(I_{K}\right)^{2 / 3}} \sqrt{\sum_{q=1}^{K} \sqrt[3]{I_{q}}} \cdot Y
$$

where $X$ and $Y$ are defined with (9).

The total distortion $D$ is obtained by summation of (18) and (19). $D$ depends on only one unknown parameter $r_{\max }$. Optimal value of $r_{\max }$ is obtained by the numerical minimization of $D$, i.e. by solving the equation $\left(d D / d r_{\max }\right)=0$. The designing process of MUPQ will be summarized in the following algorithm (recall that values of $N$ and $K$ are given in advance):

1. Optimal value of $r_{\max }$ is calculated by the numerical solving of the equation $\left(d D / d r_{\max }\right)=0$.

2. Based on (16), the optimal value of $L$ is calculated. For the practical realization, $L$ is rounded to the nearest integer divisible with $K$. This rounding has negligible effect on performances, since $K$ takes small values (usually, $4 \leq K \leq 8$ ).

3. Thresholds $r_{i}=i r_{\max } / L$ and representation levels $m_{i}=(i-1 / 2) r_{\max } / L, i=1, \ldots, L$, as well as borders between segments $t_{j}=j r_{\max } / K, j=1, \ldots, K$ are calculated.

4. Using (17), the optimal number of phase levels $M_{j}$, $j=1, \ldots, K$ for each segment is calculated. For the practical realization, values of $M_{j}$ should be rounded to the nearest integers. This rounding has negligible effect on performances.

5. Thresholds $\phi_{j, l}=l 2 \pi / M_{j}$ and representation levels $\psi_{j, l}=(l-1 / 2) 2 \pi / M_{j}, j=1, \ldots, K, l=1, \ldots, M_{j}$, are calculated, for the quantization of the phase $\phi$.

\section{Numerical Results and Discussion}

In Tab. 3, numerical results for MUPQ are presented, for different values of $N$ and $K$. Simulation of this quantizer is done in MATLAB. Let $\mathrm{SQNR}_{\text {th }}$ denote value of SQNR obtained by the theory and $\mathrm{SQNR}_{\text {sim }}$ denote value of SQNR obtained by the simulation. We can see that 


\begin{tabular}{|c|c|c|c|c|c|}
\hline $\begin{array}{c}\mathrm{R} \\
{[\mathrm{bps}]}\end{array}$ & $K$ & $r_{\max }$ & $L$ & $\begin{array}{c}\mathrm{SQNR}_{\text {th }} \\
{[\mathrm{dB}]}\end{array}$ & $\begin{array}{c}\mathrm{SQNR}_{\text {sim }} \\
{[\mathrm{dB}]}\end{array}$ \\
\hline \multirow{6}{*}{6} & 3 & 3.87 & 45 & 31.65 & 31.65 \\
\cline { 2 - 6 } & 4 & 3.90 & 44 & 31.79 & 31.77 \\
\cline { 2 - 6 } & 5 & 3.92 & 45 & 31.87 & 31.85 \\
\cline { 2 - 6 } & 6 & 3.94 & 48 & 31.89 & 31.90 \\
\cline { 2 - 6 } & 7 & 3.95 & 49 & 31.90 & 31.87 \\
\cline { 2 - 6 } & 8 & 3.96 & 48 & 31.95 & 31.93 \\
\hline \multirow{6}{*}{7} & 3 & 4.24 & 93 & 37.43 & 37.41 \\
\cline { 2 - 6 } & 4 & 4.28 & 96 & 37.60 & 37.58 \\
\cline { 2 - 6 } & 5 & 4.31 & 95 & 37.7 & 37.72 \\
\cline { 2 - 6 } & 6 & 4.33 & 96 & 37.76 & 37.74 \\
\cline { 2 - 6 } & 7 & 4.35 & 98 & 37.80 & 37.77 \\
\cline { 2 - 6 } & 8 & 4.36 & 96 & 37.83 & 37.79 \\
\hline \multirow{4}{*}{8} & 3 & 4.59 & 195 & 43.28 & 43.25 \\
\cline { 2 - 5 } & 4 & 4.64 & 200 & 43.48 & 43.48 \\
\cline { 2 - 5 } & 5 & 4.67 & 205 & 43.60 & 43.62 \\
\cline { 2 - 5 } & 6 & 4.69 & 210 & 43.67 & 43.67 \\
\cline { 2 - 5 } & 7 & 4.71 & 210 & 43.72 & 43.71 \\
\cline { 2 - 5 } & 8 & 4.73 & 208 & 43.75 & 43.77 \\
\hline
\end{tabular}

Tab. 3. Numerical results for MUPQ.

values of SQNR obtained by the theory and by the simulation are matched very well, which proves the previously developed theory.

Comparison between MUPQ and UUPQ is presented in Tab. 4 (values of SQNR $_{\text {th }}$ for UUPQ are taken from Tab. 1). From Tab. 4 we can see that SQNR increases with the increasing of $K$, becoming closer to SQNR of UUPQ. On the other hand, the increasing of $K$ leads to the increasing of complexity. Therefore, optimal choice of $K$ should be made, taking into account both SQNR and complexity. We propose values $K=5,6$ and 7 as very good solutions.

In the aim of completeness, the comparison between MUPQ and PUPQ (product uniform polar quantizer) is also presented in Tab. 4. PUPQ is well known in literature [5], [6]; this is the polar quantizer where the uniform quantization of magnitude is performed (as well as in MUPQ and UUPQ), but in PUPQ the number of phase levels is the same for all magnitude levels, i.e. $P_{1}=P_{2}=\ldots=P_{\mathrm{L}}=P$. PUPQ is the simplest of those three quantizers (PUPQ, UUPQ, MUPQ) since only one parameter $P$ has to be stored and calculated, but it has the smallest SQNR. MUPQ is slightly more complex than PUPQ (since we have to calculate and store $K$ parameters, but $K$ is very small number, less than 8), but MUPQ achieves higher SQNR compared to PUPQ. Slight increase of complexity provides appreciable increasing of SQNR.

Let's consider three quantizers: UUPQ, PUPQ and MUPQ with $K=7$ segments, for the same bit-rate of $R=8$ bps. UUPQ achieves SQNR of $43.90 \mathrm{~dB}$ and it requires calculation and storage of $L=219$ different values of $P_{i}$ (see Tab. 1). PUPQ achieves SQNR of $42.80 \mathrm{~dB}$ and it requires calculation and storage of one value $P$. MUPQ achieves SQNR of $43.72 \mathrm{~dB}$ and it requires calculation and storage of only $K=7$ different values of $M_{j}$. We can see that MUPQ achieves SQNR which is very close to SQNR of UUPQ (decreasing of SQNR is only $0.18 \mathrm{~dB}$ ), while

\begin{tabular}{|c|c|c|c|c|c|c|c|}
\hline \multirow[b]{2}{*}{$\begin{array}{c}\mathrm{R} \\
\text { [bps] }\end{array}$} & \multicolumn{3}{|c|}{ MUPQ } & \multicolumn{2}{|c|}{ UUPQ } & \multicolumn{2}{|c|}{ PUPQ } \\
\hline & $K$ & $\begin{array}{c}\mathrm{SQNR}_{\text {th }} \\
{[\mathrm{dB}]}\end{array}$ & $\begin{array}{c}\mathrm{SQNR}_{\mathrm{e}} \\
{[\mathrm{dB}]}\end{array}$ & $\begin{array}{c}\mathrm{SQNR}_{\text {th }} \\
\text { [dB] }\end{array}$ & $\begin{array}{c}\text { SQNR }_{\mathrm{e}} \\
{[\mathrm{dB}]}\end{array}$ & $\begin{array}{l}\mathrm{SQNR}_{\text {th }} \\
{[\mathrm{dB}]}\end{array}$ & $\begin{array}{c}\mathrm{SQNR}_{\mathrm{e}} \\
{[\mathrm{dB}]}\end{array}$ \\
\hline \multirow{6}{*}{6} & 3 & 31.65 & 31.75 & \multirow{6}{*}{32.06} & \multirow{6}{*}{32.21} & \multirow{6}{*}{31.37} & \multirow{6}{*}{31.27} \\
\hline & 4 & 31.79 & 31.86 & & & & \\
\hline & 5 & 31.87 & 31.96 & & & & \\
\hline & 6 & 31.89 & 32.04 & & & & \\
\hline & 7 & 31.90 & 32.13 & & & & \\
\hline & 8 & 31.95 & 32.10 & & & & \\
\hline \multirow{6}{*}{7} & 3 & 37.43 & 37.40 & \multirow{6}{*}{37.95} & \multirow{6}{*}{38.10} & \multirow{6}{*}{37.06} & \multirow{6}{*}{36.83} \\
\hline & 4 & 37.60 & 37.69 & & & & \\
\hline & 5 & 37.70 & 37.73 & & & & \\
\hline & 6 & 37.76 & 37.83 & & & & \\
\hline & 7 & 37.80 & 37.90 & & & & \\
\hline & 8 & 37.83 & 37.88 & & & & \\
\hline \multirow{6}{*}{8} & 3 & 43.28 & 43.28 & \multirow{6}{*}{43.90} & \multirow{6}{*}{44.21} & \multirow{6}{*}{42.80} & \multirow{6}{*}{42.58} \\
\hline & 4 & 43.48 & 43.65 & & & & \\
\hline & 5 & 43.60 & 43.80 & & & & \\
\hline & 6 & 43.67 & 43.90 & & & & \\
\hline & 7 & 43.72 & 43.94 & & & & \\
\hline & 8 & 43.75 & 43.93 & & & & \\
\hline
\end{tabular}

Tab. 4. Comparison of MUPQ with UUPQ and PUPQ and experimental results.

MUPQ is much simpler for realization since it requires calculation and storage of drastically smaller number of parameters (7 instead of 219). Therefore, MUPQ is much better solution than UUPQ. On the other hand, MUPQ is slightly more complex than PUPQ (7 parameters are calculated and stored instead of 1) but SQNR of MUPQ is appreciable higher (for $0.92 \mathrm{~dB}$ ) than SQNR of PUPQ. Therefore, MUPQ is better solution than PUPQ.

One application scenario is also considered, i.e. an experiment is performed applying developed theory on the speech signal. We choose the speech signal since it can be modeled very well with the Gaussian distribution [1]. Experimentally obtained values of SQNR are presented in Tab. $4\left(\mathrm{SQNR}_{\mathrm{e}}\right)$. We can see two things. Firstly, experimental results are matched well with the theoretical results, which proves the developed theory. Secondly, experimental results confirm our previous conclusion: SQNR of MUPQ is very close to SQNR of UUPQ; on the other hand, SQNR of MUPQ is appreciable higher than SQNR of PUPQ.

MUPQ can be considered as a generalized uniform polar quantizer, whose special cases are UUPQ (for $K=L$ ) and PUPQ (for $K=1$ ). The aim of MUPQ is to achieve the best ratio between SQNR and complexity, i.e. to achieve SQNR near to SQNR of UUPQ and to achieve complexity near to complexity of PUPQ.

\section{Conclusion}

The main goal of this paper is the design of the multiproduct uniform polar quantizer (MUPQ), using the asymptotic analysis. It has been known from the literature that the unrestricted uniform polar quantizer (UUPQ) could achieve very good performances, much better than the scalar uniform quantizer, but it is very complex for realiza- 
tion since large number of parameters should be calculated and stored in memory. Therefore, in this paper we proposed the multiproduct uniform polar quantizer which can achieve performances very close to performances of UUPQ, while it is much simpler for realization than UUPQ. It was shown that MUPQ is also better solution than PUPQ. The aim of MUPQ is to achieve the best ratio between SQNR and complexity. MUPQ can be used for any signal with the Gaussian distribution (a lot of real signals belong to this category).

\section{Acknowledgements}

This paper is supported by the Serbian Ministry of Science, projects TR-32045 and TR-32035.

\section{References}

[1] JAYANT, N. S., NOLL, P. Digital Coding of Waveforms. New Jersey: Prentice-Hall, 1984.

[2] GERSHO, A., GRAY, R. M. Vector Quantization and Signal Compression. Massachusetts: Kluwer Academic Publishers, 1992.

[3] SWASZEK, P., KU, T. Asymptotic performances of unrestricted polar quantizer. IEEE Transactions on Information Theory, 1986, vol. 32, no. 2, p. 330-333. DOI: 10.1109/TIT.1986.1057141

[4] NEUHOFF, D. Polar quantization revisited. In Proceedings of IEEE International Symposium on Information Theory. Ulm (Germany), 1997, p. 60. DOI: 10.1109/ISIT.1997.612975

[5] SWASZEK, P. Uniform spherical coordinate quantization of spherically symmetric sources. IEEE Transactions on Communications, 1985, vol. 33, no. 6, p. 518-521. DOI: 10.1109/TCOM.1985.1096333

[6] MOO, P. W., NEUHOFF, D. Uniform polar quantization revisited. In Proceedings of IEEE International Symposium on Information Theory ISIT'98. Cambridge (USA), 1998. DOI: 10.1109/ISIT.1998.708687

[7] HAMKINS, J., ZEGER, K. Gaussian source coding with spherical codes. IEEE Transactions on Information Theory, 2002, vol. 48, no. 11 , p. 2980-2989. DOI: 10.1109/TIT.2002.804056

[8] RAVELLI, E., DAUDET, L. Embedded polar quantization. IEEE Signal Processing Letters, 2007, vol. 14, no. 10, p. 657-660. DOI: 10.1109/LSP.2007.896379

[9] MATSCHKAL, B., HUBER, J. B. Spherical logarithmic quantization. IEEE Transactions on Audio, Speech and Language Processing, 2010, vol. 18, no. 1, p. 126-140. DOI: 10.1109/TASL.2009.2024383
[10] DINCIC, M., PERIC, Z., PETKOVIC, M., DENIC, D. Design of product polar quantizers for $\mathrm{A} / \mathrm{D}$ conversion of measurement signals with Gaussian distribution. Measurement, 2013, vol. 46, no. 8 , p. $2441-2446$

[11] DINCIC, M., PERIC, Z. Log-polar quantizer with embedded the ITU-T G.711 codec. Radioengineering, 2010, vol. 19, no. 4, p. 712-717.

[12] PERIC, Z., DINCIC, M., PETKOVIC, M. The general design of asymptotic unrestricted polar quantizers with square cells. Digital Signal Processing, 2013, vol. 23, no. 5, p. 1731-1737. DOI: $10.1016 /$ j.dsp.2013.06.001

[13] PERIC, Z., STEFANOVIC, M. Asymptotic analysis of optimal uniform polar quantization. International Journal of Electronics and Communications $A E U, 2002$, vol. 56, no. 5, p. 345-347. DOI:10.1078/1434-8411-54100111

[14] StOjanovic, D., AleKsic, D., PERIC, Z., JOVANOVIC, A. Analysis of uniform polar quantization over Bennett's integral. Elektronika ir elektrotechnika, 2005, vol. 8(64), p. 5-9.

[15] JOVANOVIC, A., PERIC, Z. Geometric piecewise uniform lattice vector quantization of the memoryless Gaussian source. Information Sciences, 2011, vol. 181, no. 14, p. 3043-3053. DOI: $10.1016 /$ j.ins.2011.03.012

\section{About the Authors ...}

Milan DINCIC was born in Nis, Serbia, in 1983. He received the B. S. degree from the Faculty of Electronic Engineering, Nis, Serbia, in 2007, and Ph.D. degree from the University of Nis, in 2012. His current research interests include the information theory, signal processing and compression, scalar and vector quantization. $\mathrm{He}$ is an author of about 20 papers in digital communications.

Zoran PERIC was born in Nis, Serbia, in 1964. He received the B. Sc., M. Sc. and Ph. D. from the Faculty of Electronic Engineering, University of Nis, Serbia, in 1989, 1994 and 1999, respectively. He is currently a Full Professor at the Department of Telecommunications and vicedean of the Faculty of Electronic Engineering Nis. His current research interests include the information theory, source and channel coding and signal processing. He is particularly working on scalar and vector quantization techniques in speech and image coding. He is an author and coauthor of over 170 papers in digital communications. Dr Peric has been a Reviewer for IEEE Transactions on Information Theory, IEEE Transactions on Signal Processing, Informatica, Information Technology and Control, Electronics and Electrical Engineering and The International Journal for Computation and Mathematics in Electrical Engineering (COMPEL). 